Supporting Information for

\title{
Fast T-type Photochromic Crystals of Diarylbenzene
}

Shota Hamatani, Daichi Kitagawa*, and Seiya Kobatake*

Department of Applied Chemistry, Graduate School of Engineering, Osaka City University, 3-3-

138 Sugimoto, Sumiyoshi-ku, Osaka 558-8585, Japan

*E-mail: kitagawa@osaka-cu.ac.jp; kobatake@a-chem.eng.osaka-cu.ac.jp 


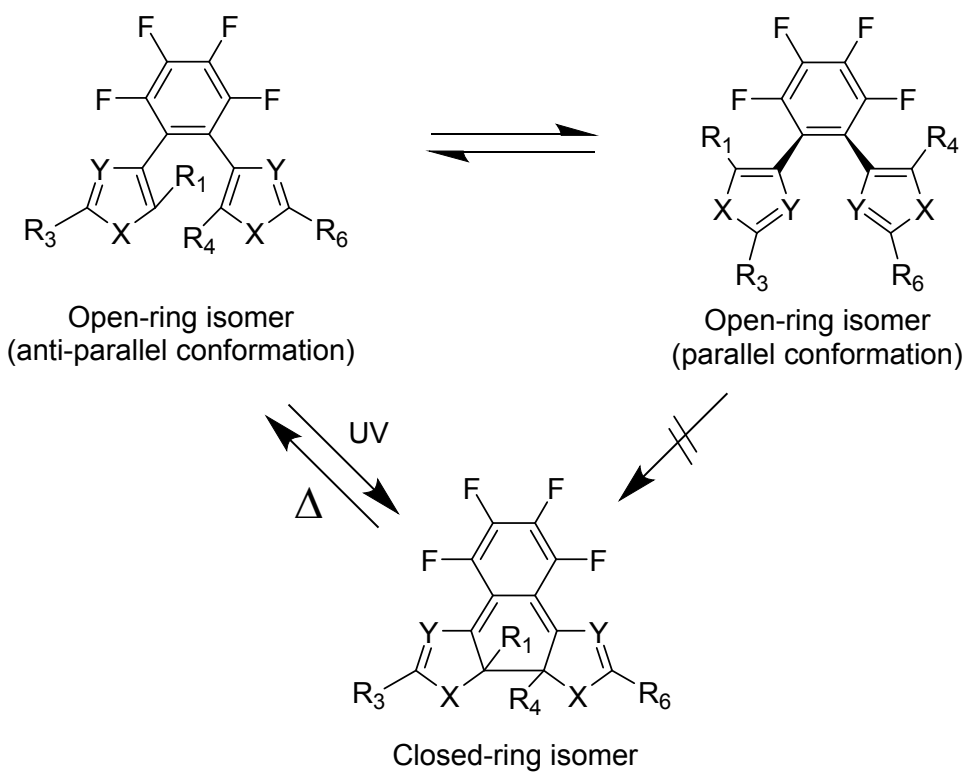

Figure S1. Two conformations of diarylbenzene open-ring isomer with two aryl moieties in mirror symmetry and $C 2$ symmetry, which are called parallel and anti-parallel conformations. Photochromic reaction undergoes only from anti-parallel conformation when the distance between reactive carbons is shorter than $4.0 \AA$.
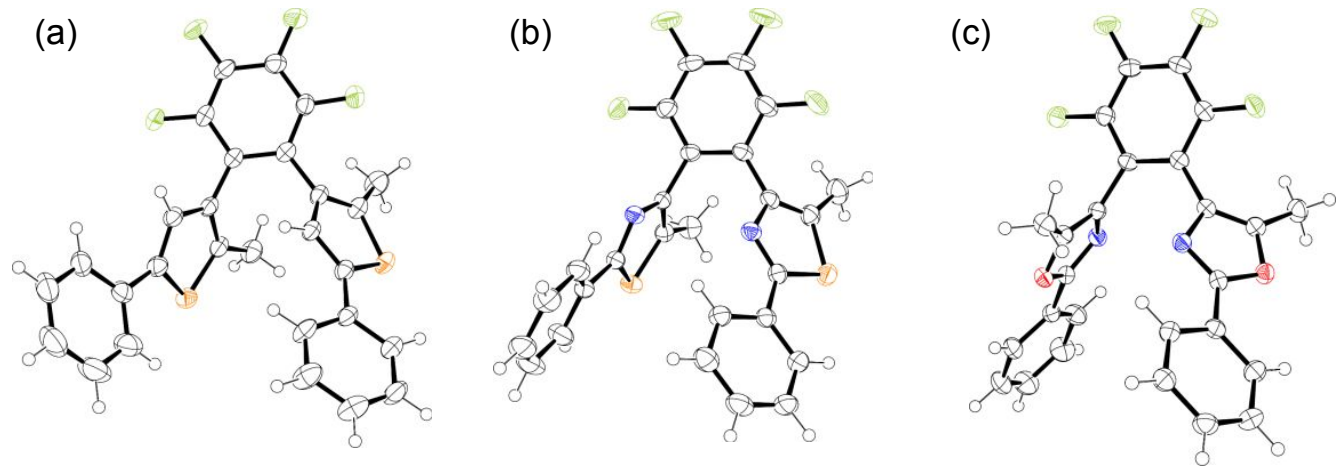

Figure S2. ORTEP drawings (showing 50\% probability displacement ellipsoids) of the openring isomer in (a) crystal 1a, (b) crystal 2a, and (c) crystal 3a. The open-ring isomer in crystal 1a and 2a was fixed in the non-photoreactive parallel conformation. The open-ring isomer in crystal 3a was fixed in anti-parallel conformation, but the distance between reactive carbons was 4.813(3) $\AA$. 


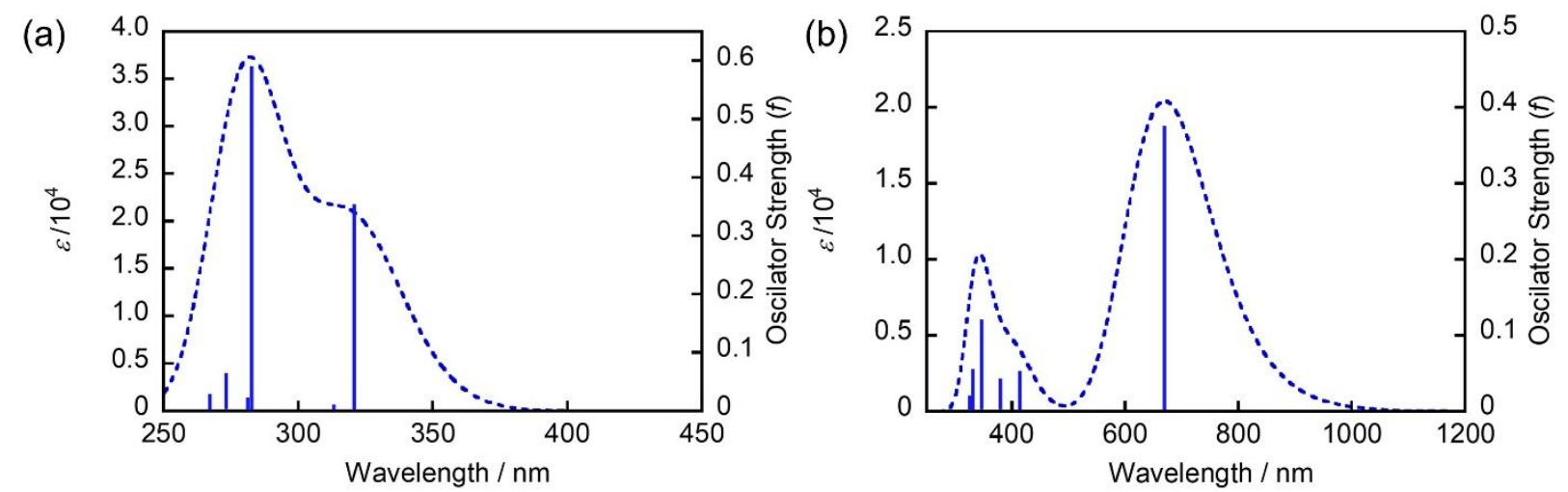

Figure S3. Calculated absorption spectra of (a) 4a and (b) $4 \mathbf{b}$ by TD-DFT (MPW1PW91/631G(d)//M06-2X/6-31G(d) level of the theory), modeled with a half width at half-maximum (HWHM) of $2000 \mathrm{~cm}^{-1}$.

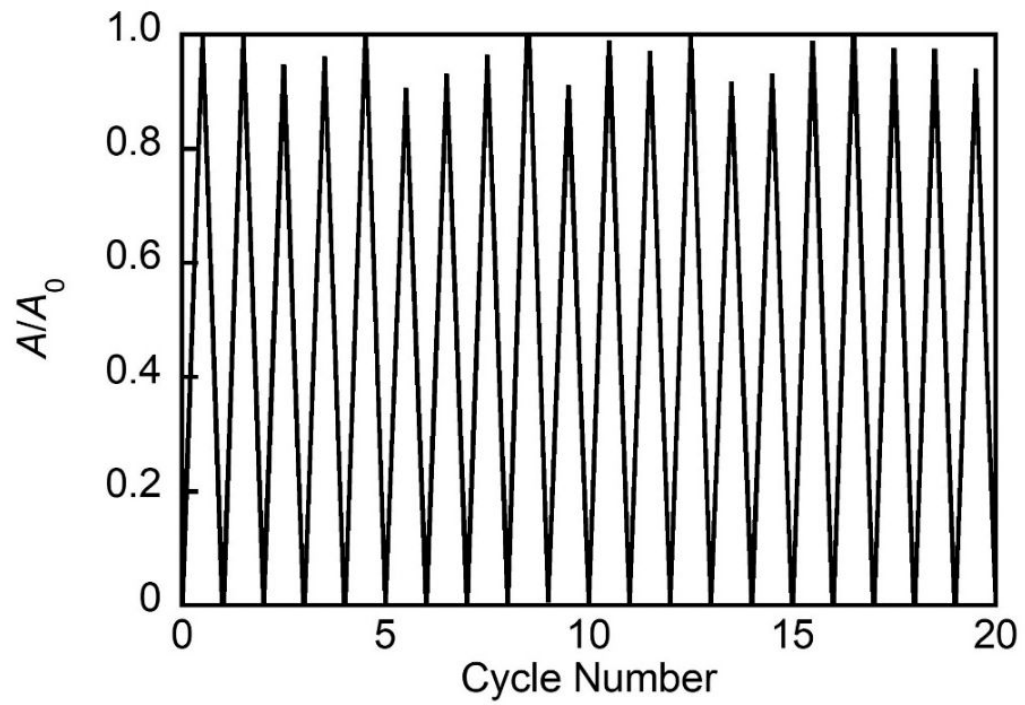

Figure S4. Repeated cycles upon alternating photoirradiation at $313 \mathrm{~nm}\left(120 \mathrm{~mW} \mathrm{~cm}{ }^{-2}\right)$ for $1.5 \mathrm{~s}$ and thermal back reaction at $318 \mathrm{~K}$. 

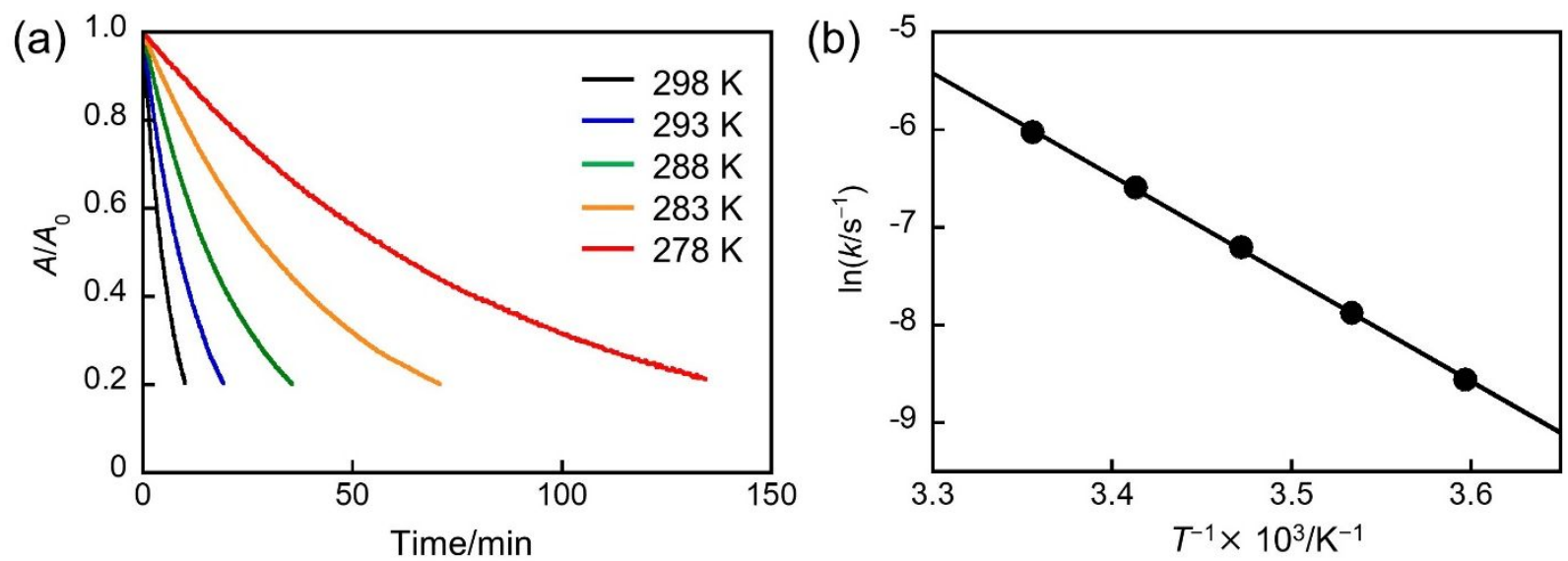

Figure S5. (a) Absorption decay curves at $\lambda_{\max }$ for $\mathbf{4 b}$ in $n$-hexane at various temperatures and (b) temperature dependence of the rate constant $(k)$ for thermal back reaction of $\mathbf{4 b}$.

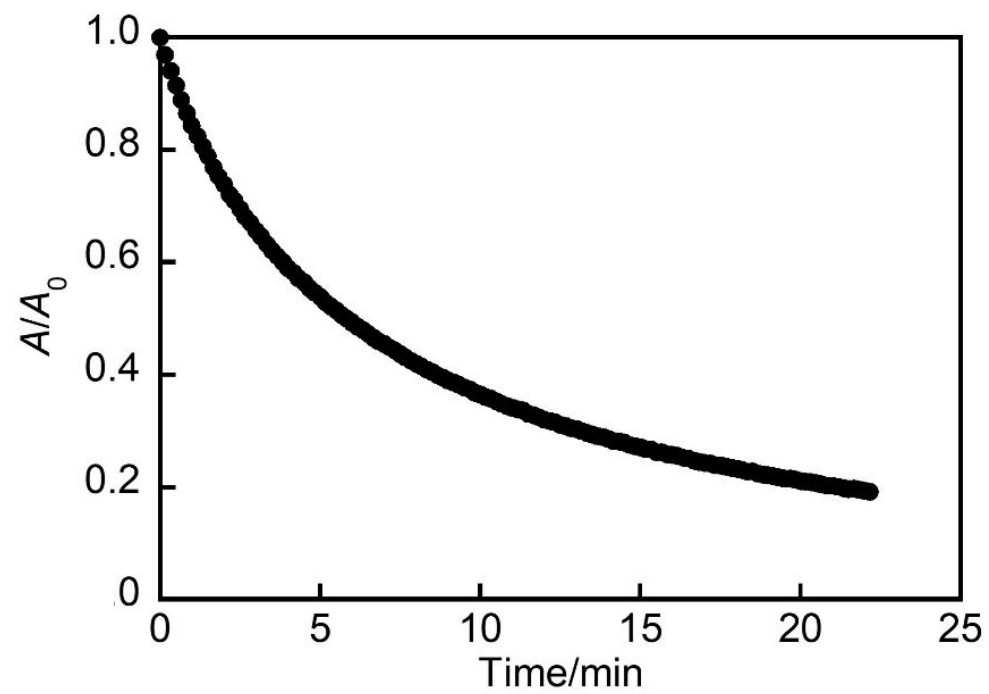

Figure S6. Absorption decay curve of $\mathbf{4 b}$ monitored at $690 \mathrm{~nm}$ in PMMA film doped with $4 \mathrm{wt} \%$ of $\mathbf{4 a}$ at $298 \mathrm{~K}$. 


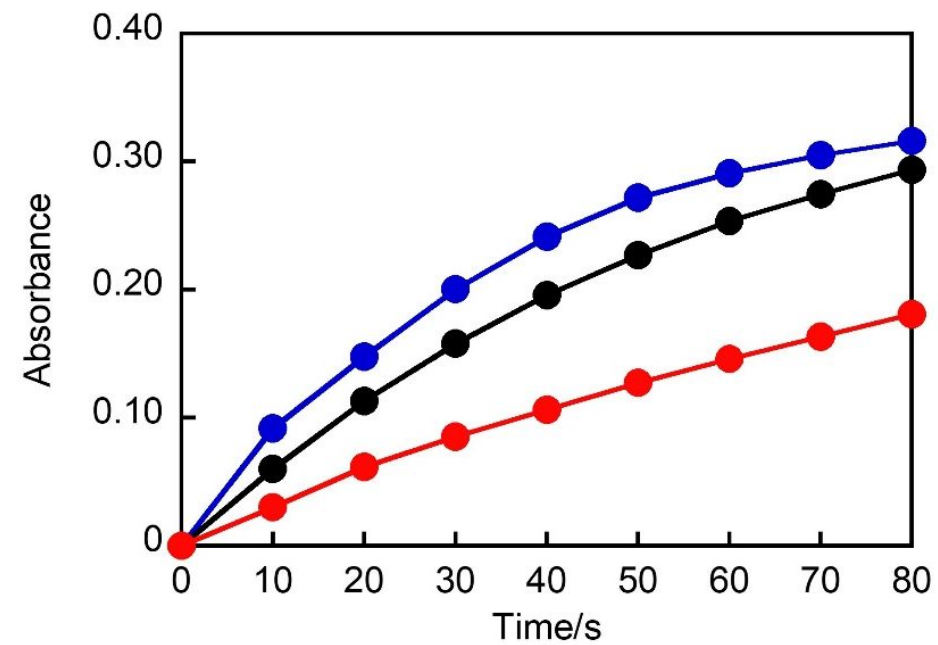

Figure S7. Absorption changes at $\lambda_{\max }$ of $\mathbf{1 b}$ (red circle), $\mathbf{2 b}$ (black circle) and $\mathbf{4 b}$ (blue circle) upon irradiation with $313 \mathrm{~nm}$ light $\left(1.5 \mathrm{~mW} \mathrm{~cm}^{-2}\right)$ at $193 \mathrm{~K}$. The initial solutions of 1a, 2a and 4a have the same absorbance at $313 \mathrm{~nm}\left(\mathrm{Abs}_{313 \mathrm{~nm}}=0.5\right)$.

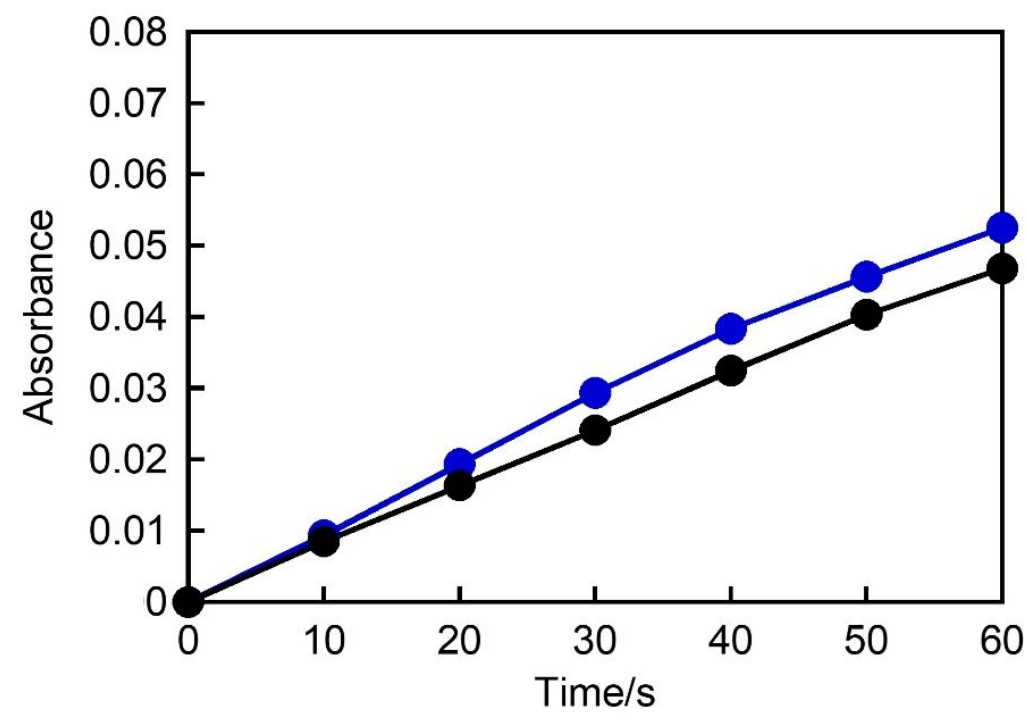

Figure S8. Absorption changes at $\lambda_{\max }$ of $\mathbf{2 b}$ (black circle) and $\mathbf{4 b}$ (blue circle) in methanol upon irradiation with $313 \mathrm{~nm}$ light $\left(2.0 \mathrm{~mW} \mathrm{~cm}^{-2}\right)$ at $228 \mathrm{~K}$. The initial solutions of $2 \mathbf{a}$ and $4 \mathbf{a}$ have the same absorbance at $313 \mathrm{~nm}\left(\mathrm{Abs}_{313 \mathrm{~nm}}=0.3\right)$. 


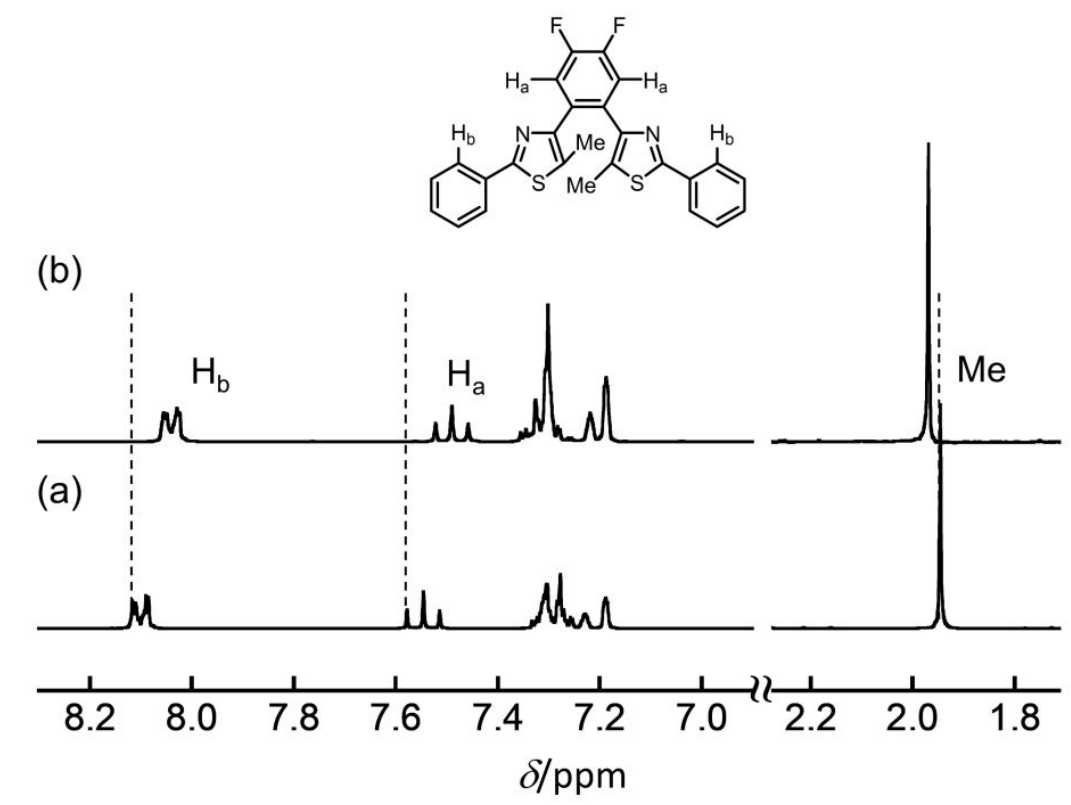

Figure S9. ${ }^{1} \mathrm{H}$ NMR spectra of $\mathbf{4 a}$ in (a) toluene- $d 8$ and (b) mixed solvent of toluene- $d 8$ and methanol-d4 (5:1).

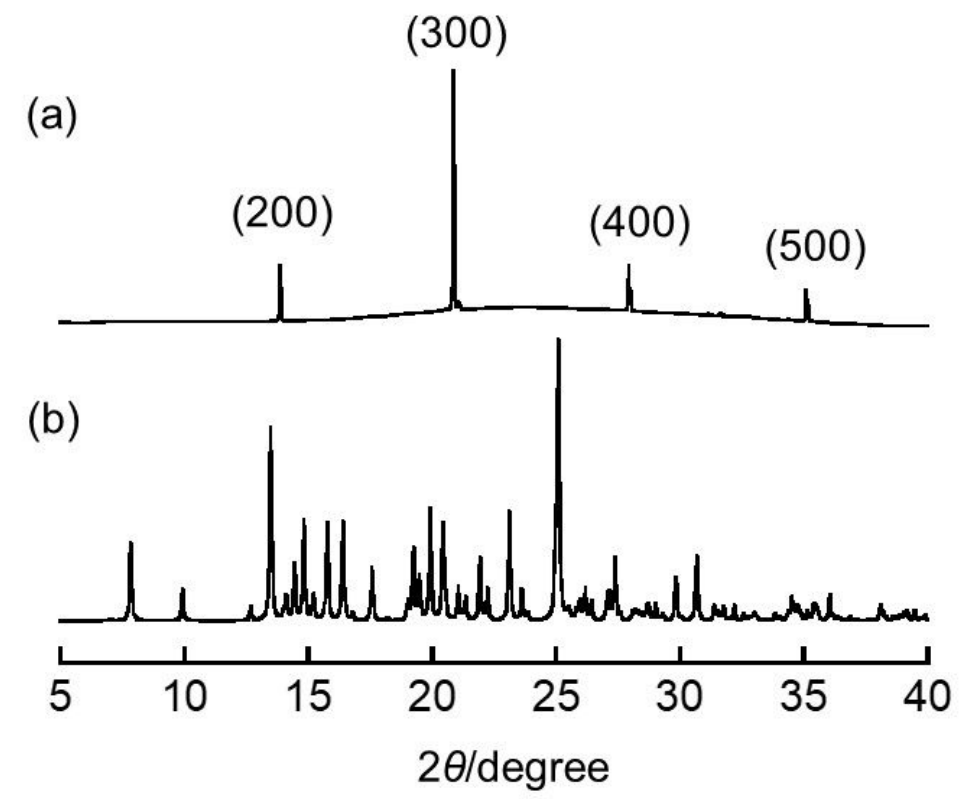

Figure S10. (a) PXRD pattern of the broadest face for crystal 4a and (b) the pattern calculated from single crystal X-ray crystallographic data of $\mathbf{4 a}$. 


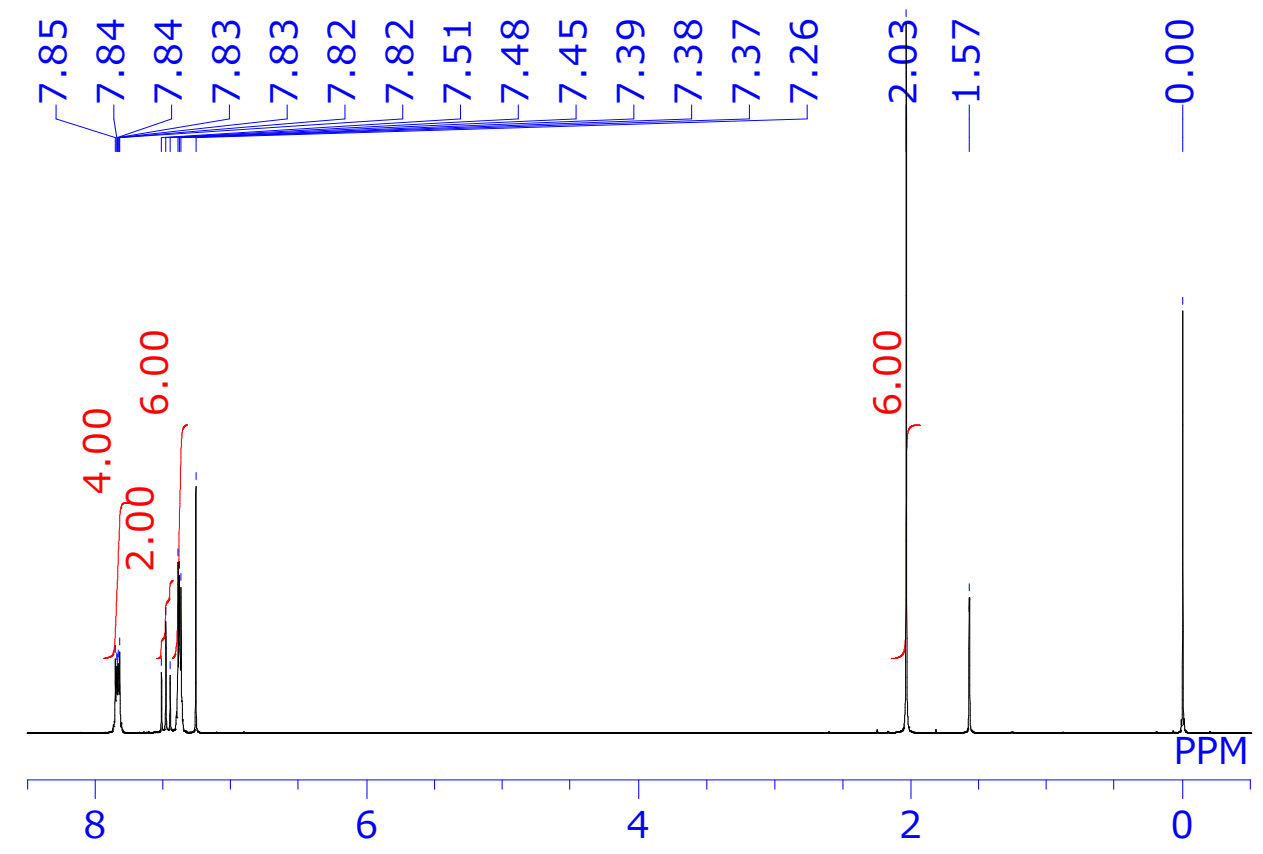

Figure S11. ${ }^{1} \mathrm{H}$ NMR spectrum of $\mathbf{4 a}$ in $\mathrm{CDCl}_{3}$.

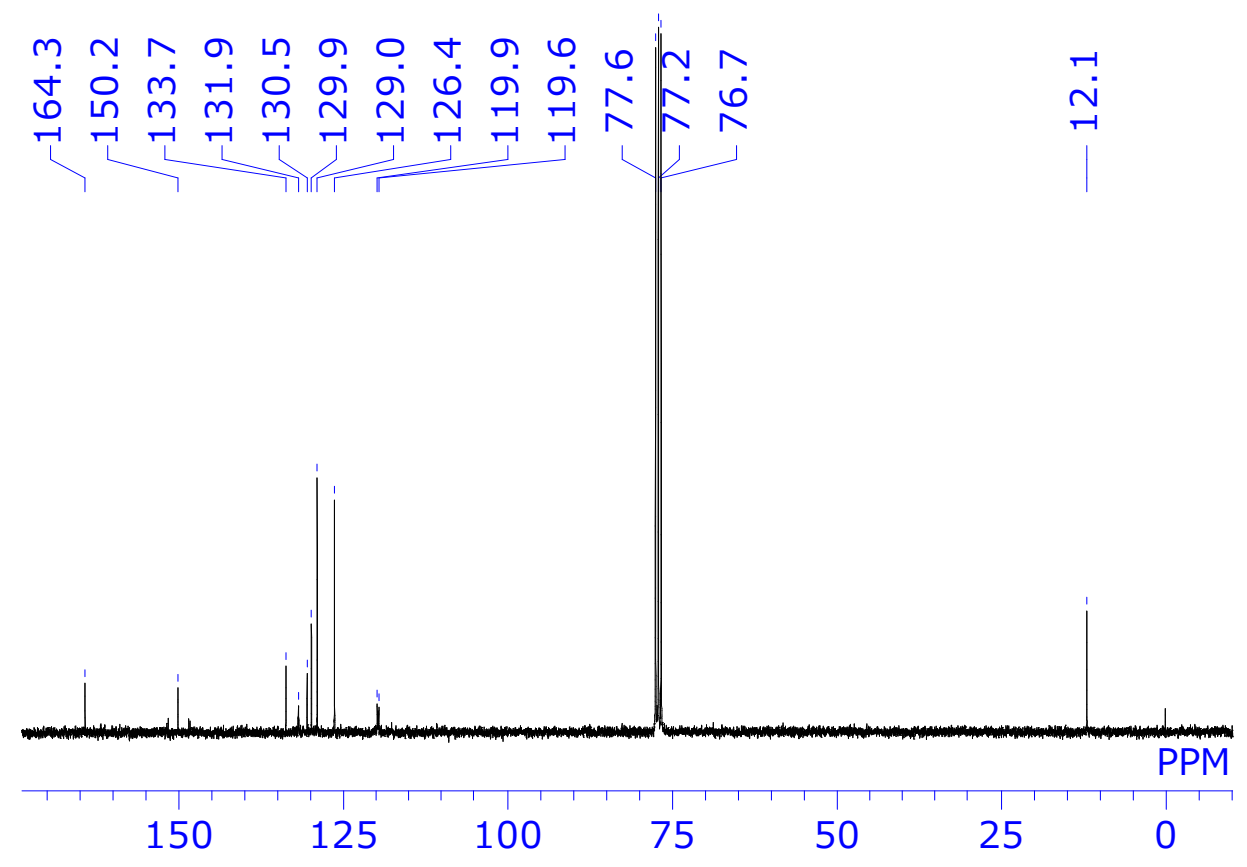

Figure S12. ${ }^{13} \mathrm{C}$ NMR spectrum of $4 \mathbf{a}$ in $\mathrm{CDCl}_{3}$. 


\section{Kinetic analysis of thermal back reaction}

The reaction kinetics of the thermal back reaction was analyzed as follows: If the thermal back reaction from the closed-ring isomer to the open-ring isomer obey a first-order kinetics, the kinetic equation is expressed as following equation by using Lambert-Beer law.

$$
\ln \frac{A_{t}}{A_{0}}=-k t
$$

where $k$ is reaction rate constant, $t$ is reaction time, and $A_{0}$ and $A_{\mathrm{t}}$ are absorbance of the closedring isomer at initial state $(t=0 \mathrm{~s})$ and at arbitrary reaction time $t$, respectively. The $k$ value can be calculated from the slope of the linear plot. The calculated $k$ values are summarized in Table S1 and S2.

Arrhenius equation can be described as follows.

$$
\ln k=\ln A-\frac{E_{a}}{R T}
$$

where $A$ is frequency factor, $E_{\mathrm{a}}$ is activation energy, $R$ is gas constant, and $T$ is absolute temperature. The linear relationship can be obtained by plotting $\ln k$ relative to $1 / T$ as shown in Figure S4 and Figure 8. The $E_{\mathrm{a}}$ and $A$ values can be determined from the slope and intercept of the linear plot. The results are summarized in Table 2 and Table 3.

Table S1. First-order rate constants for the thermal back reaction in $n$-hexane of $\mathbf{4 b}$.

\begin{tabular}{ll}
\hline$T / \mathrm{K}$ & $k / \mathrm{s}^{-1}$ \\
\hline 298 & 0.002451 \\
293 & 0.001368 \\
288 & 0.000746 \\
283 & 0.000382
\end{tabular}


Table S2. First-order rate constants for the thermal back reaction in crystal of $\mathbf{4 b}$.

\begin{tabular}{ll}
\hline$T / \mathrm{K}$ & $\mathrm{k} / \mathrm{s}^{-1}$ \\
\hline 300 & 0.364486 \\
303 & 0.749012 \\
308 & 1.228389 \\
313 & 1.698789 \\
318 & 2.143704 \\
\hline
\end{tabular}

Table S3. X-ray crystallographic data for $1 \mathrm{a}-\mathbf{4 a}$.

\begin{tabular}{|c|c|c|c|c|}
\hline & $1 \mathbf{a}^{a}$ & $2 \mathbf{a}$ & $3 a$ & $4 a$ \\
\hline Formula & $\mathrm{C}_{28} \mathrm{H}_{18} \mathrm{~F}_{4} \mathrm{~S}_{2}$ & $\mathrm{C}_{26} \mathrm{H}_{16} \mathrm{~F}_{4} \mathrm{~N}_{2} \mathrm{~S}_{2}$ & $\mathrm{C}_{26} \mathrm{H}_{16} \mathrm{~F}_{4} \mathrm{~N}_{2} \mathrm{O}_{2}$ & $\mathrm{C}_{26} \mathrm{H}_{18} \mathrm{~F}_{2} \mathrm{~N}_{2} \mathrm{~S}_{2}$ \\
\hline Formula weight & 494.54 & 496.53 & 464.41 & 460.564 \\
\hline Temperature/K & $150(2)$ & $150(2)$ & $150(2)$ & $120(2)$ \\
\hline Crystal system & Orthorhombic & Orthorhombic & Orthorhombic & Monoclinic \\
\hline Space group & $P 2_{1} 2_{1} 2_{1}$ & $P 2_{1} 2_{1} 2_{1}$ & $\mathrm{Pca}_{1}$ & $P 2_{1} / c$ \\
\hline$a / \AA$ & $7.5411(3)$ & $7.5367(5)$ & $23.488(4)$ & $12.6491(2)$ \\
\hline$b / \AA$ & $14.0447(5)$ & $12.1564(8)$ & $13.488(3)$ & $25.0889(5)$ \\
\hline$c / \AA$ & $22.0416(9)$ & $24.9857(16)$ & $6.8185(12)$ & $6.7996(2)$ \\
\hline$\alpha / \operatorname{deg}$ & 90 & 90 & 90 & 90 \\
\hline$\beta / \operatorname{deg}$ & 90 & 90 & 90 & $90.0296(19)$ \\
\hline$\gamma / \operatorname{deg}$ & 90 & 90 & 90 & 90 \\
\hline Volume $/ \AA^{3}$ & $2334.48(16)$ & $2289.2(3)$ & $2160.1(7)$ & $2157.87(8)$ \\
\hline$Z$ & 4 & 4 & 4 & 4 \\
\hline Density $/ \mathrm{g} \mathrm{cm}^{-3}$ & 1.407 & 1.441 & 1.428 & 1.418 \\
\hline Goodness-of-fit on $F^{2}$ & 1.066 & 1.080 & 1.056 & 1.024 \\
\hline$R(I>2 \sigma(I))$ & $R_{1}=0.0372$ & $R_{1}=0.0343$ & $R_{1}=0.0303$ & $R_{1}=0.0360$ \\
\hline$R$ (all data) & $w R 2=0.0909$ & $w R 2=0.0774$ & $w R 2=0.0722$ & $w R 2=0.0983$ \\
\hline CCDC No. & 1872163 & 2044772 & 2044771 & 2044770 \\
\hline
\end{tabular}


Table S4. Oscillator strength $(f)$ of $\mathbf{4}$ calculated by time-dependent density functional theory (TD-DFT) at the MPW1PW91/6-31G(d)//M06-2X/6-31G(d) level.

\begin{tabular}{|c|c|c|c|}
\hline \multicolumn{2}{|c|}{$4 a$} & \multicolumn{2}{|c|}{$4 b$} \\
\hline$\lambda / \mathrm{nm}$ & $f$ & $\lambda / \mathrm{nm}$ & $f$ \\
\hline 321.0 & 0.352 & 669.8 & 0.374 \\
\hline 313.2 & 0.004 & 413.7 & 0.052 \\
\hline 282.6 & 0.583 & 380.8 & 0.041 \\
\hline 281.2 & 0.018 & 347.1 & 0.121 \\
\hline 273.2 & 0.065 & 331.8 & 0.058 \\
\hline 267.4 & 0.031 & 327.5 & 0.015 \\
\hline
\end{tabular}

Table S5. Cartesian coordinates of 4a optimized at the M06-2X/6-31G(d) level.

\begin{tabular}{|c|c|c|c|}
\hline Atom & $\mathrm{X} / \AA$ & $\mathrm{Y} / \AA$ & $Z / \AA$ \\
\hline $\mathrm{C}$ & 0.656737 & 1.724561 & 0.249855 \\
\hline $\mathrm{C}$ & -0.65674 & 1.724563 & -0.24986 \\
\hline $\mathrm{C}$ & -1.31416 & 2.944653 & -0.45711 \\
\hline $\mathrm{C}$ & -0.66014 & 4.135272 & -0.2213 \\
\hline $\mathrm{C}$ & 0.660147 & 4.13527 & 0.221303 \\
\hline $\mathrm{C}$ & 1.314167 & 2.944649 & 0.457108 \\
\hline $\mathrm{C}$ & 1.396614 & 0.485788 & 0.589567 \\
\hline $\mathrm{C}$ & 3.217099 & -0.77649 & 0.496457 \\
\hline $\mathrm{C}$ & -1.39662 & 0.485794 & -0.58958 \\
\hline $\mathrm{C}$ & -3.2171 & -0.77648 & -0.49646 \\
\hline $\mathrm{S}$ & 2.153592 & -1.76334 & 1.472221 \\
\hline
\end{tabular}




\begin{tabular}{|c|c|c|c|}
\hline $\mathrm{S}$ & -2.15361 & -1.76331 & -1.47226 \\
\hline $\mathrm{C}$ & 0.92785 & -0.54653 & 1.359156 \\
\hline $\mathrm{C}$ & -0.92786 & -0.54652 & -1.35917 \\
\hline $\mathrm{C}$ & -0.39044 & -0.7077 & 2.048347 \\
\hline $\mathrm{H}$ & -0.25756 & -1.0918 & 3.063795 \\
\hline $\mathrm{H}$ & -0.90392 & 0.255231 & 2.105873 \\
\hline $\mathrm{H}$ & -1.04402 & -1.40069 & 1.505038 \\
\hline $\mathrm{C}$ & 0.390437 & -0.70769 & -2.04836 \\
\hline $\mathrm{H}$ & 0.257557 & -1.0918 & -3.06381 \\
\hline $\mathrm{H}$ & 0.903911 & 0.255233 & -2.10589 \\
\hline $\mathrm{H}$ & 1.044012 & -1.40069 & -1.50505 \\
\hline $\mathrm{F}$ & 1.271111 & 5.305006 & 0.426846 \\
\hline $\mathrm{F}$ & -1.2711 & 5.30501 & -0.42684 \\
\hline $\mathrm{C}$ & -4.57411 & -1.20987 & -0.12707 \\
\hline $\mathrm{C}$ & -5.10806 & -2.41851 & -0.58331 \\
\hline $\mathrm{C}$ & -5.34388 & -0.39084 & 0.707366 \\
\hline $\mathrm{C}$ & -6.38988 & -2.80548 & -0.20988 \\
\hline $\mathrm{H}$ & -4.52315 & -3.06346 & -1.23455 \\
\hline $\mathrm{C}$ & -6.62466 & -0.78103 & 1.077077 \\
\hline $\mathrm{H}$ & -4.9185 & 0.544446 & 1.05565 \\
\hline $\mathrm{C}$ & -7.15166 & -1.98846 & 0.621548 \\
\hline $\mathrm{H}$ & -6.79356 & -3.74626 & -0.57053 \\
\hline
\end{tabular}




\begin{tabular}{|c|c|c|c|}
\hline $\mathrm{H}$ & -7.21547 & -0.14075 & 1.72479 \\
\hline $\mathrm{H}$ & -8.15249 & -2.29096 & 0.913352 \\
\hline $\mathrm{C}$ & 4.574112 & -1.20987 & 0.127079 \\
\hline $\mathrm{C}$ & 5.108056 & -2.41853 & 0.583306 \\
\hline $\mathrm{C}$ & 5.343889 & -0.39084 & -0.70734 \\
\hline $\mathrm{C}$ & 6.389879 & -2.80548 & 0.209894 \\
\hline $\mathrm{H}$ & 4.523131 & -3.06348 & 1.234526 \\
\hline $\mathrm{C}$ & 6.624681 & -0.78102 & -1.07704 \\
\hline $\mathrm{H}$ & 4.918523 & 0.544457 & -1.05561 \\
\hline $\mathrm{C}$ & 7.151666 & -1.98846 & -0.62152 \\
\hline $\mathrm{H}$ & 6.793551 & -3.74627 & 0.570531 \\
\hline $\mathrm{H}$ & 7.215495 & -0.14073 & -1.72473 \\
\hline $\mathrm{H}$ & 8.152502 & -2.29095 & -0.91331 \\
\hline $\mathrm{N}$ & -2.68683 & 0.349742 & -0.12527 \\
\hline $\mathrm{N}$ & 2.686831 & 0.349737 & 0.125266 \\
\hline $\mathrm{H}$ & -2.33708 & 2.961868 & -0.81731 \\
\hline $\mathrm{H}$ & 2.337088 & 2.96186 & 0.817309 \\
\hline
\end{tabular}

Table S6. Cartesian coordinates of $\mathbf{4 b}$ optimized at the M06-2X/6-31G(d) level.

\begin{tabular}{cccc}
\hline Atom & $\mathrm{X} / \AA$ & $\mathrm{Y} / \AA$ & $\mathrm{Z} / \AA$ \\
\hline $\mathrm{C}$ & -0.00678 & -0.7349 & 1.764836 \\
$\mathrm{C}$ & 0.006776 & 0.734896 & 1.764836
\end{tabular}




\begin{tabular}{|c|c|c|c|}
\hline $\mathrm{C}$ & -0.06336 & 1.43197 & 3.030091 \\
\hline $\mathrm{C}$ & -0.03816 & 0.723398 & 4.1716 \\
\hline $\mathrm{C}$ & 0.038157 & -0.7234 & 4.1716 \\
\hline $\mathrm{C}$ & 0.063359 & -1.43197 & 3.030091 \\
\hline $\mathrm{C}$ & -0.11821 & -1.40215 & 0.576919 \\
\hline $\mathrm{C}$ & -0.01399 & -3.15103 & -0.78196 \\
\hline $\mathrm{C}$ & 0.118212 & 1.402152 & 0.576919 \\
\hline $\mathrm{C}$ & 0.013994 & 3.151029 & -0.78196 \\
\hline $\mathrm{S}$ & 0.055971 & -1.85918 & -2.01484 \\
\hline $\mathrm{S}$ & -0.05597 & 1.859183 & -2.01484 \\
\hline $\mathrm{C}$ & -0.41452 & -0.6433 & -0.71723 \\
\hline $\mathrm{C}$ & 0.414524 & 0.643296 & -0.71723 \\
\hline $\mathrm{C}$ & -1.93646 & -0.40747 & -0.78691 \\
\hline $\mathrm{H}$ & -2.44743 & -1.36553 & -0.66333 \\
\hline $\mathrm{H}$ & -2.25132 & 0.266496 & 0.015957 \\
\hline $\mathrm{H}$ & -2.22673 & 0.025871 & -1.74582 \\
\hline $\mathrm{C}$ & 1.936458 & 0.40747 & -0.78691 \\
\hline $\mathrm{H}$ & 2.447432 & 1.365526 & -0.66333 \\
\hline $\mathrm{H}$ & 2.251315 & -0.2665 & 0.015957 \\
\hline $\mathrm{H}$ & 2.226725 & -0.02587 & -1.74582 \\
\hline $\mathrm{F}$ & 0.071245 & -1.30551 & 5.371963 \\
\hline $\mathrm{F}$ & -0.07125 & 1.305508 & 5.371963 \\
\hline
\end{tabular}




\begin{tabular}{|c|c|c|c|}
\hline $\mathrm{C}$ & -0.03052 & 4.562376 & -1.18043 \\
\hline $\mathrm{C}$ & -0.03574 & 4.941085 & -2.52698 \\
\hline $\mathrm{C}$ & -0.07125 & 5.550108 & -0.18609 \\
\hline $\mathrm{C}$ & -0.08189 & 6.286163 & -2.87552 \\
\hline $\mathrm{H}$ & 0.006424 & 4.184568 & -3.3056 \\
\hline $\mathrm{C}$ & -0.11599 & 6.890841 & -0.53938 \\
\hline $\mathrm{H}$ & -0.06774 & 5.241931 & 0.853849 \\
\hline $\mathrm{C}$ & -0.12179 & 7.262823 & -1.88399 \\
\hline $\mathrm{H}$ & -0.0835 & 6.57103 & -3.92276 \\
\hline $\mathrm{H}$ & -0.14704 & 7.651129 & 0.234626 \\
\hline $\mathrm{H}$ & -0.15735 & 8.313054 & -2.15675 \\
\hline $\mathrm{C}$ & 0.030515 & -4.56238 & -1.18043 \\
\hline $\mathrm{C}$ & 0.035742 & -4.94109 & -2.52698 \\
\hline $\mathrm{C}$ & 0.071245 & -5.55011 & -0.18609 \\
\hline $\mathrm{C}$ & 0.081888 & -6.28616 & -2.87552 \\
\hline $\mathrm{H}$ & -0.00642 & -4.18457 & -3.3056 \\
\hline $\mathrm{C}$ & 0.11599 & -6.89084 & -0.53938 \\
\hline $\mathrm{H}$ & 0.067739 & -5.24193 & 0.853849 \\
\hline $\mathrm{C}$ & 0.12179 & -7.26282 & -1.88399 \\
\hline $\mathrm{H}$ & 0.083496 & -6.57103 & -3.92276 \\
\hline $\mathrm{H}$ & 0.147036 & -7.65113 & 0.234626 \\
\hline $\mathrm{H}$ & 0.157353 & -8.31305 & -2.15675 \\
\hline
\end{tabular}




$\begin{array}{cccc}\mathrm{N} & -0.08189 & -2.76986 & 0.449157 \\ \mathrm{~N} & 0.081887 & 2.769864 & 0.449157 \\ \mathrm{H} & -0.09637 & 2.51468 & 3.045793 \\ \mathrm{H} & 0.096374 & -2.51468 & 3.045793\end{array}$

Table S7. Cartesian coordinates of transition state of 4 optimized at the M06-2X/6-31G(d) level.

\begin{tabular}{|c|c|c|c|}
\hline Atom & $\mathrm{X} / \AA \AA$ & $\mathrm{Y} / \AA$ & $Z / \AA$ \\
\hline $\mathrm{C}$ & -0.34834 & -0.61637 & 1.861988 \\
\hline $\mathrm{C}$ & 0.348344 & 0.616374 & 1.861988 \\
\hline $\mathrm{C}$ & 0.671402 & 1.22644 & 3.071764 \\
\hline $\mathrm{C}$ & 0.329775 & 0.611159 & 4.265681 \\
\hline $\mathrm{C}$ & -0.32978 & -0.61116 & 4.265681 \\
\hline $\mathrm{C}$ & -0.6714 & -1.22644 & 3.071764 \\
\hline $\mathrm{C}$ & -0.6748 & -1.26175 & 0.579212 \\
\hline $\mathrm{C}$ & -0.6748 & -2.97492 & -0.82304 \\
\hline $\mathrm{C}$ & 0.674801 & 1.261748 & 0.579212 \\
\hline $\mathrm{C}$ & 0.674801 & 2.974915 & -0.82304 \\
\hline $\mathrm{S}$ & -0.98124 & -1.65698 & -1.94997 \\
\hline $\mathrm{S}$ & 0.981244 & 1.656979 & -1.94997 \\
\hline $\mathrm{C}$ & -0.85147 & -0.46985 & -0.62851 \\
\hline $\mathrm{C}$ & 0.851465 & 0.469847 & -0.62851 \\
\hline $\mathrm{C}$ & -1.89528 & 0.630739 & -0.69156 \\
\hline
\end{tabular}




\begin{tabular}{|c|c|c|c|}
\hline $\mathrm{H}$ & -2.89429 & 0.18975 & -0.64561 \\
\hline $\mathrm{H}$ & -1.78477 & 1.319889 & 0.149402 \\
\hline $\mathrm{H}$ & -1.80978 & 1.198482 & -1.62267 \\
\hline $\mathrm{C}$ & 1.895276 & -0.63074 & -0.69156 \\
\hline $\mathrm{H}$ & 2.894289 & -0.18975 & -0.64561 \\
\hline $\mathrm{H}$ & 1.784774 & -1.31989 & 0.149402 \\
\hline $\mathrm{H}$ & 1.809784 & -1.19848 & -1.62267 \\
\hline $\mathrm{F}$ & -0.63458 & -1.1812 & 5.433629 \\
\hline $\mathrm{F}$ & 0.634575 & 1.181204 & 5.433629 \\
\hline $\mathrm{C}$ & 0.536957 & 4.359729 & -1.24877 \\
\hline $\mathrm{C}$ & 0.639774 & 4.73035 & -2.59703 \\
\hline $\mathrm{C}$ & 0.297772 & 5.352918 & -0.28456 \\
\hline $\mathrm{C}$ & 0.505001 & 6.060893 & -2.972 \\
\hline $\mathrm{H}$ & 0.824243 & 3.975141 & -3.3569 \\
\hline $\mathrm{C}$ & 0.167858 & 6.679864 & -0.66622 \\
\hline $\mathrm{H}$ & 0.216882 & 5.058879 & 0.756281 \\
\hline $\mathrm{C}$ & 0.270076 & 7.040768 & -2.00988 \\
\hline $\mathrm{H}$ & 0.584957 & 6.334009 & -4.01955 \\
\hline $\mathrm{H}$ & -0.0148 & 7.439258 & 0.087786 \\
\hline $\mathrm{H}$ & 0.166985 & 8.08016 & -2.30522 \\
\hline $\mathrm{C}$ & -0.53696 & -4.35973 & -1.24877 \\
\hline $\mathrm{C}$ & -0.63977 & -4.73035 & -2.59703 \\
\hline
\end{tabular}




\begin{tabular}{|c|c|c|c|}
\hline $\mathrm{C}$ & -0.29777 & -5.35292 & -0.28456 \\
\hline $\mathrm{C}$ & -0.505 & -6.06089 & -2.972 \\
\hline $\mathrm{H}$ & -0.82424 & -3.97514 & -3.3569 \\
\hline $\mathrm{C}$ & -0.16786 & -6.67986 & -0.66622 \\
\hline $\mathrm{H}$ & -0.21688 & -5.05888 & 0.756281 \\
\hline $\mathrm{C}$ & -0.27008 & -7.04077 & -2.00988 \\
\hline $\mathrm{H}$ & -0.58496 & -6.33401 & -4.01955 \\
\hline $\mathrm{H}$ & 0.014802 & -7.43926 & 0.087786 \\
\hline $\mathrm{H}$ & -0.16699 & -8.08016 & -2.30522 \\
\hline $\mathrm{N}$ & -0.55382 & -2.58387 & 0.431613 \\
\hline $\mathrm{N}$ & 0.553821 & 2.58387 & 0.431613 \\
\hline $\mathrm{H}$ & -1.19632 & -2.17538 & 3.089482 \\
\hline $\mathrm{H}$ & 1.196319 & 2.175375 & 3.089482 \\
\hline
\end{tabular}

